\title{
Translation of Japanese Noun Compounds at Super-Function Based MT System
}

\author{
Xin Zhao Non-member (The University of Tokushima) \\ Fuji Ren Non-member (The University of Tokushima, ren@is.tokushima-u.ac.jp) \\ Shingo Kuroiwa Member (The University of Tokushima, kuroiwa@is.tokushima-u.ac.jp)
}

Keywords: Noun Compounds, NLP, Machine Translation, SFBMT, Monolingual corpus

In this paper, we propose a noun compounds translation method for Japanes to Chinese Machine Translation (MT) systems. The translation of noun compounds has become a major issue in MT due to their frequency of occurrence and high productivity, especially in Japanese. In our previous studies on Super-Function Based Machine Translation (SFBMT), we have found that noun compounds are very frequently used and difficult to be translated correctly. Especially, the overgeneration of noun compounds can be dangerous as it may introduce much ambiguity in the translation.

We discuss in this paper the challenges in handling Japanese noun compounds in an SFBMT system. Fig. 1 shows a flowchart of the proposed noun compounds translation module for SFBMT system. The proposed method uses a shallow method for translating noun compounds by using a word level translation dictionary and target language monolingual corpus. It is much easier to obtain a

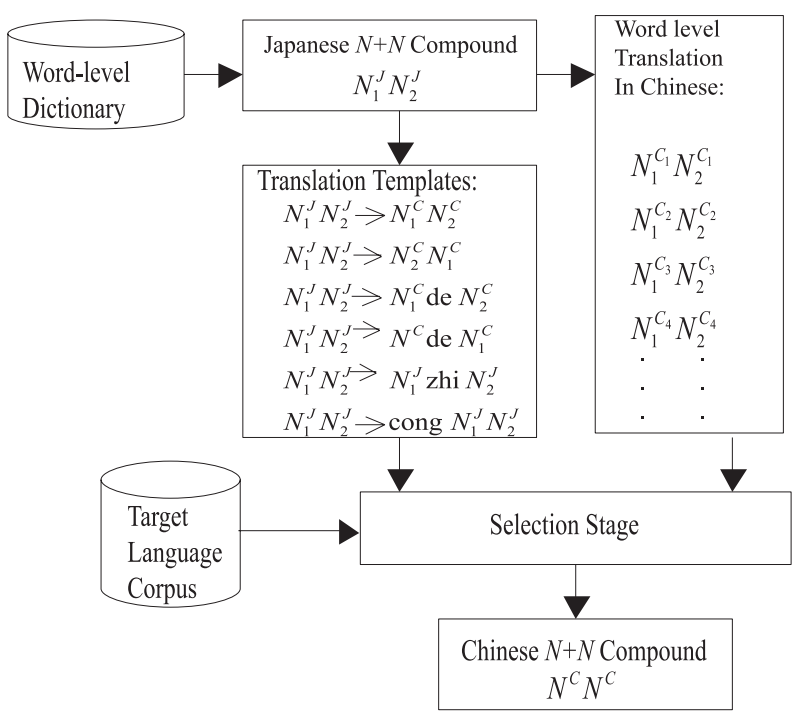

Fig. 1. Frowchart of the propsed method large size of monolingual corpus than bilingual corpus, so that we can get accurate compound nouns translation rules. We developed this noun compound translation method as a standalone module for SFBMT sytem.

We conducted Japanese-Chinese MT experiments using sentences that included noun compounds. First, we evaluated our proposed template-based word-alignment method. Table 1 shows the experimental results. Partially aligned case in the table shows the case where some nouns are lost from a compound noun. Next, we conducted noun compounds translation experiments using correctly aligned Japanese sentences in the first experiment. Table 2 shows the experimental results. From these results, although the problem still remains in the word-alignment method, we can conclude that the translating method is effective for noun compounds translation.

Table 1. Results of compound noun alignment

\begin{tabular}{rrr}
\hline \hline Results & Number & Coverage \\
\hline \hline Correctly aligned & 54 & $68 \%$ \\
\hline Partially aligned & 19 & $24 \%$ \\
\hline Unaligned & 5 & $6 \%$ \\
\hline No template found & 2 & $2 \%$ \\
\hline \hline
\end{tabular}

Table 2. Result of compound noun translation

\begin{tabular}{llrc}
\hline \hline No. & Template & \multicolumn{2}{c}{ Accuracy } \\
\hline \hline 1. & $N_{1}^{J} N_{2}^{J} \rightarrow N_{1}^{C} N_{2}^{C}$ & $94 \%$ & $(30 / 32)$ \\
\hline 2. & $N_{1}^{J} N_{2}^{J} \rightarrow N_{2}^{C} N_{1}^{C}$ & $80 \%$ & $(12 / 15)$ \\
\hline 3. & $N_{1}^{J} N_{2}^{J} \rightarrow N_{1}^{C} d e N_{2}^{C}$ & $67 \%$ & $(2 / 3)$ \\
\hline 4. & $N_{1}^{J} N_{2}^{J} \rightarrow N_{2}^{C} d e N_{1}^{C}$ & $100 \%$ & $(1 / 1)$ \\
\hline 5. & $N_{1}^{J} N_{2}^{J} \rightarrow N_{2}^{C} l e N_{1}^{C}$ & - & $(0 / 0)$ \\
\hline 6. & $N_{1}^{J} N_{2}^{J} \rightarrow N_{1}^{C} z h i N_{2}^{C}$ & $100 \%$ & $(2 / 2)$ \\
\hline 7. & $N_{1}^{J} N_{2}^{J} \rightarrow \operatorname{cong} N_{1}^{C} N_{2}^{C}$ & $100 \%$ & $(1 / 1)$ \\
\hline \hline Total & & $89 \%$ & $(48 / 54)$ \\
\hline \hline
\end{tabular}




\title{
Translation of Japanese Noun Compounds at Super-Function Based MT System
}

\author{
Xin Zhao* \\ Fuji Ren* \\ Non-member \\ Shingo Kuroiwa* Member
}

Noun compounds are frequently encountered construction in nature language processing (NLP), consisting of a sequence of two or more nouns which functions syntactically as one noun. The translation of noun compounds has become a major issue in Machine Translation (MT) due to their frequency of occurrence and high productivity. In our previous studies on Super-Function Based Machine Translation (SFBMT), we have found that noun compounds are very frequently used and difficult to be translated correctly, the overgeneration of noun compounds can be dangerous as it may introduce ambiguity in the translation. In this paper, we discuss the challenges in handling Japanese noun compounds in an SFBMT system, we present a shallow method for translating noun compounds by using a word level translation dictionary and target language monolingual corpus.

Keywords: Noun Compounds, NLP, Machine Translation, SFBMT, Monolingual corpus

\section{Introduction}

Machine translation (MT) describes computer-based translation between human languages and is one of the oldest large-scale applications of computer science. There is an increasing demand for MT systems which produce high quality translations and which can be easily adapted to many language pairs, new domains and changing terminology.

Multiword expressions are a key problem for the development of large-scale, linguistically natural language processing technology due to the idiomaticity and overgeneration problems. Idiomaticity is the problem of compositional semantic unpredictability and/or syntactic markedness. Overgeneration occurs as a result of a system failing to capture idiosyncratic lexical affinities between word combinations. Noun compounds, especially noun-noun compounds are very frequently used in some languages such as Japanese, Chinese, English, etc., and are often important portion determining the semantic content of the document. These noun compounds are created from different combinations of nouns and are too large in number to be contained in a manuallycreated dictionary, thus automatic acquisition of their translations is highly desirable. The translation of noun compounds has become a major issue in MT due to their frequency of occurrence and high productivity. We know that noun compounds pose well-known problems for linguistic description in general as well as the problems of identification, segmentation, disambiguation, interpretation, and so on. All of those problems make them

\footnotetext{
* Faculty of Engineering, University of Tokushima

Tokushima 770-8506, Japan
}

particularly difficult to be handled in a system performing automatic translation, such as a machine translation system or a system for cross-language information retrieval.

Various claims have been made about the level of processing complexity required to translate noun compounds, and proposed translation methods range over a broad spectrum of processing complexity. Several strategies based on shallow processing have been proposed for the translation of compounds. The simplest ones rely on static transfer dictionary that list compounds, either obtained from aligned parallel corpora or by traditional lexicographic work. There is a fatal shortcoming that it is not able to cope with the productive formation of new compounds. More elaborate strategies ${ }^{(1)}$ dynamically assign translations to source language compounds. Many studies on the task of terminology translation have relied on parallel or comparable corpora ${ }^{(2)-(6)}$. However sufficient amount of such corpora are still hardly available. In contrast with them, monolingual corpora are much easily available and it would be desirable to accomplish word translation by using them alone.

In this paper, we discuss the challenges of translating Japanese noun compounds into Chinese in the SuperFunction Based Machine Translation (SFBMT) system. The core of the SFBMT uses the Super-Function (SF) approach to translate without going through syntactic and semantic analysis. The system uses a bilingual dictionary and the SF concept to produce a translation of a source text ${ }^{(7)}$. The Japanese language is agglutinative; which means it forms words by putting together basic elements, called morphemes, that retain their original forms and meanings with little change during the combination process. Our interest in noun compounds 
stemmed from the realization that they are highly frequent and highly productive in Japanese. In our previous studies on SFBMT we have found that those noun compounds are frequently used and difficult to be translated correctly. In order to enhance the performance of the system and the quality of translation, we propose a shallow solution for translating those noun compounds in SFBMT.

In Section 2 we give a brief introduction of our previous studies on SFBMT. Section 3 describe the problems of noun compounds in SFBMT and the proposed shallow method. Section 4 outlines the resources used in this research and present the results. Section 5 contextualises this research with respect to other related work. Finally, we conclude in Section 6.

\section{Super-Function Based MT System}

Super-Function (SF) is a function that shows some defined relations between a source language and a target language sentence pair ${ }^{(8)(9)}$. We assume that a sentence consists of some constant and some variable parts. By replacing the variable parts one can get a multitude of different sentences. In our system, we only look at nouns as variables for translation. That is, we can use an SF to describe the detailed relation of those nouns and the residual constant parts between a source and a target language sentence pair ${ }^{(7)}$.

2.1 Expression of Super-Function To illustrate this concept, we provide an example:

$\mathrm{SF}^{\dagger}$ :

$X_{j 1}$ NITSUITEHA $X_{j 2}$ NO $X_{j 3}$ NIGORENNRAKU KUDASAI. $\Longrightarrow$ YOUGUANYU $X_{c 1}$ QINGYU $X_{c 2}$ DE $X_{c 3}$ LIANXI.

J: SYOUSAI NITSUITEHA SISYA NO NAKAMURA NIGORENNRAKU KUDASAI.

\section{C: YOUGUANYU XIJIE QINGYU FENGONGSI DE} ZHONGCUN LIANXI.

E: For details, please contact Mr. Nakamura of branch office.

For ease of readability, in the example nouns are expressed in italic type. An SF can be represented by a Node TaBle (NTB) and an Edge TaBle (ETB). See details in Tables 1 and 2. NTB is used to store the constant parts (node parts) which are extracted from Japanese and Chinese sentence pair; and ETB is used to describe the location relationship of the nouns (edge parts) between Japanese and Chinese sentence pair.

2.2 Translation Processing In SFBMT system, we use a J/C bilingual dictionary and the SF concept to produce a sentence-by-sentence translation of a source text. An input sentence is first analyzed morphologically and then matched with the source sentence and an SF. The process of SFBMT consists of three major parts:

$\dagger$ We use some phonetic spelling instead of original Japanese and Chinese character sets. In SF, $X$ indicates the variable parts (noun parts), the number indicates the corresponding positions of the $X$, $j$ and $c$ indicate Japanese and Chinese, respectively.
Table 1. Example of a node table

\begin{tabular}{ccc}
\hline \hline No & Language-J & Language-C \\
\hline \hline 1 & $\phi$ & YOUGUAN \\
2 & NITSUITEHA & QINGYU \\
3 & NO & DE \\
4 & NIGORENRAKUKUDASAI. & LIANXI. \\
\hline \hline
\end{tabular}

Table 2. Example of an edge table

\begin{tabular}{ccc}
\hline \hline No & Location-J & Location-C \\
\hline \hline 1 & 1 & 1 \\
2 & 2 & 2 \\
3 & 3 & 3 \\
\hline \hline
\end{tabular}

(1) Morphological Analysis: The Japanese sentence is morphologically analyzed by ChaSen (a free Japanese morphological analyzer).

(2) Translation Processing: The nouns are extracted from the result of the morphological analysis. The word sequences, which consist of one word or some words, between nouns represent structure of sentences and become node parts of an SF. The corresponding Chinese node parts, that is an SF, are found in the SF database, which stores Japanese-Chinese SF pairs, by searching those Japanese node parts in the database. By using the bilingual dictionary the nouns are translated into Chinese.

(3) Morphological Agreement: Based on the order of nouns in an ETB a rearrangement of the nouns within the Chinese node parts takes place. Finally a translation sentence is outputted.

The translation corpus for our experiments in the previous work consists of 391 business letters with about 4,100 sentences of Japanese and Chinese. For evaluating the system, we have chosen a sample of 250 sentences from a different source ${ }^{(10)}$. In the 250 evaluating sentences, $183(73 \%)$ sentences have been translated successfully and 204 (81\%) sentences have an SF that is available in the SF base. The result of the experiment and our experience show that using the SF in the translation system to translate without syntactic and semantic analysis can be really effective.

\section{Intractable Problems and Proposed So- lution}

In the experiment of our previous work, due to the difficulties of translating noun compounds, the translation of them was not included as an evaluation criterion. If we can find some solution to translate noun compounds properly, it is obvious that the overall performance of the system will be increased. Seeing the detailed process of SFBMT which is illustrated above, we can see that translating noun compounds could be acted as a standalone module in the SFBMT system.

3.1 Intractable Problems of Noun Compounds Since the Chinese characters are extremely productive in their ability to generate new words, compounding plays a major role in Japanese word-formation. By combining a stock of a few thousand characters, hundreds of thou- 
sands of compound words are created. We have found noun compounds are highly frequent and highly productive in Japanese and they are a very serious problem for both MT systems and human translators. In our previous studies on SFBMT we have found that it was difficult to translate them correctly, we give some examples to illustrate the difficulties in translating:

- Lexical Divergences in Japanese and Chinese: Translation pairs where one or more pairs of component nouns does not align under exact translation, but are conceptually similar. Such as BIJINESU+BAGU ${ }^{\dagger}$ (business bag) $\rightarrow$ GONGWEN $+\mathrm{BAO}$ vs. BIJINESU+MASHIN (business machine) $\rightarrow$ SHIWU+JIQI vs. BIJINESU + SOFUTO (business software) $\rightarrow$ SHANGYONG +RUANJIAN vs. BIJINESU + GURAFIKKUSU (business graphics $) \rightarrow$ SHANGYE+TUBIAO vs. BIJINESU + DAIRI (business agency) $\rightarrow$ SHANGWU +DAILI. In the above examples we can find that the underlined word BIJINESU can have many different interpretations in Chinese.

- Existence of Non-Compositional Noun Compounds: Since the condition of compound word generation is different among languages, Japanese compound words are not always translated into Chinese compound words. For each Japanese compound, the appropriate Chinese construction must thus be determined. Such as SENMON+URIBA (special counter) $\rightarrow$ ZHUANMEN+GUITAI, which is translated most naturally into Chinese as ZHUANGUI; In this case, that ZHUANGUI can be analyzed as a two character abbreviation derived from ZHUANMEN (special) and GUITAI (counter), which aligns fully with ZHUANMEN GUITAI. Another example: MEDAMA+SYOUHIN (loss leader) $\rightarrow$ YANZHU+ SHANGPIN, which translates into Chinese should be REMENHUO. Here, REMEN does not align with MEDAMA.

- Constructional Variability in the Translations: SYOUHIN+KAKAKU (price of commodity) $\left(N_{1}^{J} N_{2}^{J}\right) \rightarrow$ SHANGPIN + JIAGE $\left(N_{1}^{C} N_{2}^{C}\right)^{\dagger \dagger}$ vs.KEIYAKU +IHAN (breach of contract) $\left(N_{1}^{J} N_{2}^{J}\right)$ $\rightarrow$ WEIFAN + HETONG $\left(N_{2}^{C} N_{1}^{C}\right)$ vs.NEHIKI+RIYUU (discount reason) $\left(N_{1}^{J} N_{2}^{J}\right) \rightarrow$ JIANGJIA+de + LIYOU $\left(N_{1}^{C} d e N_{2}^{C}\right)$. For Japanese noun compounds $\left(N_{1}^{J} N_{2}^{J}\right)$, there are many different kinds of constructions in Chinese language. Such as, $\left(N_{1}^{C} N_{2}^{C}\right),\left(N_{2}^{C} N_{1}^{C}\right),\left(N_{1}^{C} d e N_{2}^{C}\right)$.

- Mismatch in Semantic Explicitness: Sometimes one compound can has multiple interpretations, and can only be reliably interpreted in the

\footnotetext{
† With all examples of Japanese and Chinese noun compounds, we segment the compound into its component nouns through the use of the "+" symbol. Note that no such segmentation boundary is indicated in the original Japanese and Chinese.

${ }^{\dagger \dagger} N_{i}^{J}$ denotes the i-th Japanese $(\mathrm{J})$ noun $(\mathrm{N})$, and $N_{i}^{C}$ denotes a Chinese (C) noun (N) corresponding to the i-th source Japanese lexeme.
}

context. For example, KEIYAKU+NAIYOU can be translated as: HEYUE+NEIRONG, QIYUE+ NEIRONG, HETONG+NEIRONG, and so on, the semantic content explicitly described in the source noun compound is made implicit in the translation. Furthermore, integrating the translated compound into its context may also present difficult choices. One specific issue concerns potential attributes to the compound.

3.2 Proposed Shallow Solution We use the Japanese-Chinese noun + noun compound MT task as a test case for a compositional translation method which makes use of a word-level dictionary and monolingual corpus data. Much work on the similar task of terminology translation has relied on parallel or comparable corpora. We attempt to use only target language corpus evidence in the translation process, to reduce data sparseness and to carry out the method easily.

For MT systems, compound nouns are too varied to be able to pre-compile an exhaustive list of translated compound nouns. Aiming to develop a robust MT system which can translate those compound nouns, we propose a shallow solution for translating compound nouns based on Japanese-Chinese SFBMT. We translate noun compounds through the composition of word level translations and a constructional translation template. When word level translations for each component nouns are facilitated, this method becomes useful. The method can act as a standalone module in the SFBMT, translating compound nouns according to the best translation candidate produced by the method. Koehn and Knight ${ }^{(11)}$ have indicated that in the context of statistical MT, overall translation performance can improve when source language compound nouns are prescriptively translated into target language compound nouns. In this work, we discuss how to generate and select the correct translation for those noun compounds, and investigate what happens when non-compositional noun compounds are translated using a compositional method. The flowchart of noun compounds processing is show in Figure 1.

Using monolingual target language corpus may give misleading clues as to how the content of a compound is best generated. Some factors (such as the presence of modifying attributes) can guide how to choose a construction type. Tanaka and Baldwin ${ }^{(1)}$ has indicated that a content, which by itself best would be expressed as a compound, may be represented by phrasal constructions in the corpus. According to our experience on Japanese-Chinese MT, it is a very effective method that translating each noun of the compound word firstly and generating a translation for the compound word using same rules.

To translate a productively formed noun compounds, there are two basic stages: generation and selection.

- Generation Stage: At the generation stage, each component word is first translated into target word resulting in various translation results. Secondly they are applied to various translation templates generating translation candidates. Each word trans- 


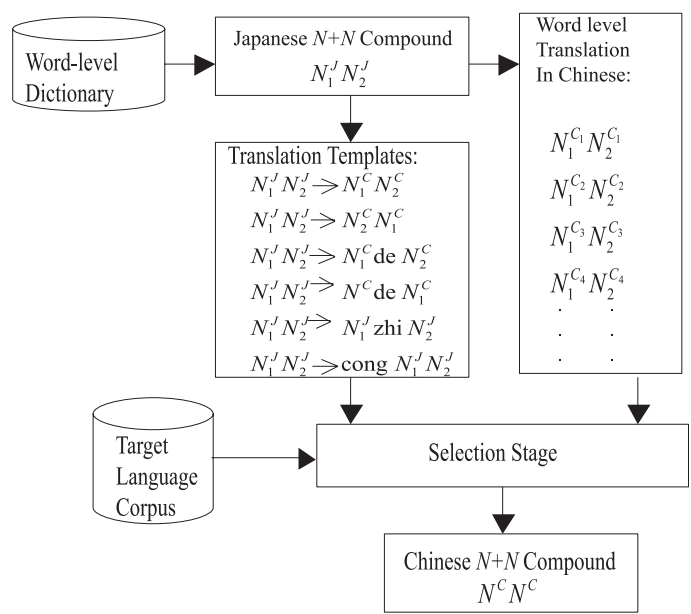

Fig. 1. Flowchart of The Processing

lation and template slot is annotated for part of speech (POS), and we constrain the generation process to output only those candidates where each slot and filler in the translation template agree in POS sort. The translation templates define possible structural mappings from source language compounds to the target language. For example, in order to translate keiyaku-ihan (breach of contract), possible translations for keiyaku (contract) are qiyue, hetong, heyue and translations for ihan (breach) are weifan, weibei, qinhai... The constructional translation template could be: $N_{1}^{J} N_{2}^{J} \rightarrow$ $N_{2}^{C} N_{1}^{C}$. Where $N_{i}^{C}$ indicats that the word is a noun (N) in Chinese (C) and corresponds to the $i$ thoccurring noun in the original Japanese, see Table 3 for further example templates. Such templates were established on the basis of a set of validated translation pair. By taking all possible combinations of the word-level translations, and applying them to the slots of templates, a set of potentially legitimate translations is generated.

Each slot in the translation template is indexed for part of speech, and derivational morphology is optionally used to convert a given word-level translation into a form appropriate for a given template. Generation fails in the instance that we are unable to find a word-level translation for $N_{1}$ and/or $N_{2}$.

- Selection Stage: In selection stage, we aim to select the most likely translation for the source noun + noun compound from the generated translation candidates. Selection is performed based on a empirical data from a monolingual target language corpus. The best translation candidate is not assumed to correspond to the most frequently occurring candidate in a target language corpus, but rather to the candidate that appears in the contexts most similar to that of the source language compound. To select the most plausible translation, the context of source language instances in monolingual corpus is compared to those of the candidate translations in a target language corpus. Each candidate is scored by interpolating probabilities of fully and partially specified translation data.

In the context of Computer Aided Translation Technology (CATT), a corpus can be described as a large collection of texts in electronic format. Electronic corpora can be "enriched" by, for example, annotating them with part-of-speech (POS) tagging, and this is especially useful in order to enable researchers to carry out sophisticated linguistic investigations. But, even an untagged corpus of texts (so-called "raw" text) can be a useful performanceenhancing tool in translating; in obtaining information about collocates (words that typically cooccur), in reinforcing knowledge of normal target language patterns, and in learning how to use new expressions. At the decision on a translation equivalent for a specific term or phrase, the corpus has been of great help in verifying or rejecting decisions based on other tools such as dictionaries and the internet. The corpus helps in deciding on which of these alternatives to use.

We take the generated translation candidates and score each, returning the highest scoring translation candidate as our final translation. Ignoring the effects of POS (part of speech) constraints for the moment, the number of generated translations is $O(m n t)$ where $m$ and $n$ are the fertility of Japanese nouns $N_{1}^{J}$ and $N_{2}^{J}$, respectively, and $t$ is the number of translation templates. As a result, there is often a large number of translation candidates to select from, and the selection method crucially determines the efficacy of the method. The scoring method rates the corpus-based translation quality of a given translation candidate according to both corpus evidence for the fully specified translation and its parts in the context of the translation template in question. This is calculated as ${ }^{\dagger}$ :

$$
\begin{aligned}
\operatorname{ctq}\left(W_{1}^{C}, W_{2}^{C}, T\right) & \\
= & \alpha p\left(W_{1}^{C}, W_{2}^{C}, T\right)+\beta p\left(W_{1}^{C}, T\right) p\left(W_{2}^{C}, T\right) \\
& +\gamma p\left(W_{1}^{C}\right) p\left(W_{2}^{C}\right) p(T) \cdots \cdots \cdots \cdots(1)
\end{aligned}
$$

$W_{1}^{C}$ and $W_{2}^{C}$ are the word level translations of the Japanese $N_{1}^{J}$ and $N_{1}^{J}$, respectively, and $T$ is the translation template ${ }^{\dagger \dagger}$. Calculating each probability according to a maximum likelihood estimate based on relative corpus occurrence, most plausible compound noun is obtained. As seen in Eq.1, the left hand side which is candidate quality is obtained as the weighted sum of probability obtained by full substitution of elements and multiplication of probability obtained by partial substitution of elements. The weights take the form of $\alpha \beta$, and $\gamma$, where $0 \leq \alpha, \beta, \gamma \leq 1$ and $\alpha+\beta+\gamma=1$. The use of linear interpolation constitutes a basic form of smoothing, in that we would like to have some means of selecting the most likely translation in the absence of evidence for the fully specified translation

\footnotetext{
$\dagger$ In the formulation, ctq means corpus-based translation quality (CTQ) (12)

${ }^{\dagger \dagger} W_{1}^{C}$ and $W_{2}^{C}$ are assumed to be POS-compatible with $T$.
} 
candidate.

In the formulation1, the product $p\left(W_{1}^{C}\right) p\left(W_{2}^{C}\right)$ $p(T)$ was included as a third term, but Tanaka and Baldwin ${ }^{(12)}$ found it to have negligible impact on translation accuracy, so we can omit it and the formulation can be:

$$
\begin{aligned}
& \operatorname{ctq}\left(W_{1}^{C}, W_{2}^{C}, T\right) \\
& \quad=\alpha p\left(W_{1}^{C}, W_{2}^{C}, T\right)+\beta p\left(W_{1}^{C}, T\right) p\left(W_{2}^{C}, T\right)
\end{aligned}
$$$$
\text { .............. (2) }
$$

In the formulation 2 , ctq is based on linear interpolation over $\alpha$ and $\beta$, where $0 \leq \alpha, \beta \leq 1$ and $\alpha+\beta=1$. We set $\alpha$ to 0.9 and $\beta$ to 0.1 throughout evaluation.

The basic intuition behind decomposing the translation candidate into its two parts within the context of the translation template $\left(p\left(W_{1}^{C}, T\right)\right.$ and $p\left(W_{2}^{C}, T\right)$ in the second term of equation 2) is to capture the subcategorisation properties of $W_{1}^{C}$ and $W_{2}^{C}$ relative to $T$. For example, if $W_{1}^{C}$ and $W_{2}^{C}$ were sheji and gaibian, respectively, and $p\left(W_{1}^{C}, W_{2}^{C}, T\right)=0$ for all $T$, we would hope to score gaibian le sheji as being more likely than sheji gaibian. We could hope to achieve this by virtue of the fact that gaibian occurs in the form gaibian le ... much more frequently, making the value of $p\left(W_{2}^{C}, T\right)$ greater for the template $N_{1}^{J} N_{2}^{J} \rightarrow$ $N_{2}^{C} l e N_{1}^{C}$ than $N_{1}^{J} N_{2}^{J} \rightarrow N_{1}^{C} N_{2}^{C}$. As a result, there is often more than one legitimate translation to select from, and the selection method crucially determines the efficacy of the solution.

\section{Resources and Evaluating Results}

We have found that noun compounds in Japanese and Chinese are really highly frequent and highly productive. In this research we proposed to translate compound nouns based on a word level translation dictionary and a target language corpus in SFBMT, and only targeted at the translation from Japanese to Chinese. In this section, we will outline the resources used in this research and present the results in details.

4.1 Used Resources To process the translation of compounds, we need some resources: a target language corpus, a word-level translation dictionary, an inventory of translation templates, and some test data which used to evaluate the method.

Corpus: In our current research, we aim to find out the solution making use of noun compounds surveyed at our previous research on SFBMT. These noun compounds consist of 391 letters with about 4,100 sentences of Chinese extracted from two books. They are Writing Business Letters In Japanese which is published by The Japan Times, Ltd. $2003^{(13)}$ and Chinese-Japanese Business Letters Encyclopedia which is published by Japan TOHO Shoten in $1995^{(14)}$.

Dictionary: The word level translation dictionary used in this research is the same as we have used in the previous study on SFBMT. We have a Japanese-English Bilingual Dictionary JEB-V015 (General vocabulary is
230,000 words), based on the dictionary, we generated a simulated Japanese-Chinese word level translation dictionary.

Translation Templates: Japanese and Chinese are unrelated languages requiring a different set of words, grammatical morphemes, and word order in sentence. The generation phase of translation relies on translation templates to recast the Japanese compound noun into Chinese. The translation templates were obtained by way of word alignment, in this research, there are 7 templates were used. They were determined by combining all conditional alignment mappings between Japanese compounds and their Chinese translations. The one most often used translation template is $N_{1}^{J} N_{2}^{J} \rightarrow N_{1}^{C} N_{2}^{C}$, one noteworthy translation template is $N_{1}^{J} N_{2}^{J} \rightarrow N_{2}^{C} N_{1}^{C}$, where the Chinese translation takes the form of an noun+noun compound but the order of the nouns is reversed. See Table 3 for details.

Test Data: In order to generate Japanese compound noun test data, first we have collected a sample of 250 sentences from a different source ${ }^{(10)}$ and segmented and tagged them by using ChaSen, and extracted out all noun + noun bigrams from it. We took only those noun + noun bigrams adjoined by non-nouns to ensure that they were not part of a larger compound nominal. The test data was selected randomly from the extracted data, which included 80 noun + noun compounds, for those compounds, any errors in segmentation were postcorrected. For the generation of a standard translation for each compound, we translated each Japanese compound into Chinese by Kingsoft PowerWord.net $2000{ }^{\dagger}$ and the word-level translation dictionary which is mentioned above. In the case that there were multiple translation candidates for a given compound, the most appropriate one was selected manually. In the case where the dictionary translations were considered to be suboptimal or inappropriate, manually obtained translation result is used. Finally accuracy of total translation result is checked.

After that we examined each Japanese-Chinese translation pair to determine if both $N_{1}^{J}$ and $N_{2}^{J}$ aligned in the Chinese translation via word-level translations. When such alignment is found, the result is classified as aligned noun + noun compound. When alignment is not found the case is classified as unaligned noun + noun compounds. We picked up translation templates concerning with successful alignment. As a result 54 compounds were classified as belonging to the aligned noun compounds, whereas 26 compound word designated as unaligned compounds.

4.2 Evaluating Results To evaluate the translation candidate scoring method, we test the effect of fluctuation of values of $\alpha$ and $\gamma$ in equation 1 , which the value of $\beta$ being determined by $1-\alpha-\gamma$, and also test its robustness over data for which translation compositionality does not hold. We evaluate translation performance according to the standard measures of

\footnotetext{
$\dagger$ A dictionary and translation software which support the translation between Chinese/Japanese/English.
} 
Table 3. Example of Translation Templates

\begin{tabular}{ll}
\hline \hline Template & Example \\
\hline \hline$N_{1}^{J} N_{2}^{J} \rightarrow N_{1}^{C} N_{2}^{C}$ & KEIYAKU+SYORUI (contract document) $\rightarrow$ HETONG+WENSHU \\
$N_{1}^{J} N_{2}^{J} \rightarrow N_{2}^{C} N_{1}^{C}$ & RIKAI+ZOUSHIN (improve undertanding) $\rightarrow$ ZENGJIN+LIAOJIE \\
$N_{1}^{J} N_{2}^{J} \rightarrow N_{1}^{C} d e N_{2}^{C}$ & KEIHI+ZOU (expenditure increase) $\rightarrow$ JINGFEI+de+ZENGJIA \\
$N_{1}^{J} N_{2}^{J} \rightarrow N_{2}^{C} d e N_{1}^{C}$ & HIYOU+FUTAN (cost burden) $\rightarrow$ FUDAN+de+FEIYONG \\
$N_{1}^{J} N_{2}^{J} \rightarrow N_{2}^{C} l e N_{1}^{C}$ & SEKKEI+HENKOU (design changes) $\rightarrow$ GAIBIAN+le+SHEJI \\
$N_{1}^{J} N_{2}^{J} \rightarrow N_{1}^{C} z h i N_{2}^{C}$ & KAIIN+AIDA (between members) $\rightarrow$ HUIYUAN+zhi+JIAN \\
$N_{1}^{J} N_{2}^{J} \rightarrow$ cong $N_{1}^{C} N_{2}^{C}$ & TOUJITSU+KISAN (reckoning from that day) $\rightarrow$ cong+DANGRI+QISUAN \\
\hline \hline
\end{tabular}

Table 4. Results of Alignment

\begin{tabular}{rcc}
\hline \hline Case & Number & Coverage \\
\hline \hline Aligned & 54 & $67.5 \%$ \\
\hline Partially Aligned & 19 & $23.7 \%$ \\
\hline Unaligned & 5 & $6.3 \%$ \\
\hline No Template Found & 2 & $2.5 \%$ \\
\hline \hline
\end{tabular}

precision, recall and F-score. Precision value is the relative proportion of inputs for which we generate a correct translation against the translation data set treated, recall is the relative proportion of inputs for which we are able to generate a translation, and F-score is the harmonic mean of the two. We evaluate our method against two baselines derived from ctq. The first baseline takes the most probable fully-specified translation candidate (i.e. is equivalent to setting $\alpha=1, \beta=0$ and $\gamma=0$ in equation 1 ). The second baseline scores translation candidates according to template-specified partial translation probabilities (i.e. is equivalent to setting $\alpha=0$, $\beta=1$ and $\gamma=0$ in equation 1 ). Baseline- 1 is prone to low recall as a result of there being no fully-specified translation candidate attested in the corpus. Baseline2 is prone to low precision as it does not consider the lexical affinity between the word-level translations.

We translated Japanese noun compounds by using the simulated Japanese-Chinese word level translation dictionary. Recall that we generate the standard Chinese translations by using this simulated Japanese-Chinese word level translation dictionary and Kingsoft PowerWord.net 2000. The standard translations were manually selected when there were multiple dictionary entries. A translation is manually generated when a more appropriate translation was considered to exist, or in the case that no translation was found. We compared word-alignment of Japanese noun compounds with their translations using the extracted translation templates. The alignment results are shown in Table 4 .

(1) Aligned: All component words of a noun compound are aligned according to one of the translation templates. Among them 54 (67.5\%) were classified as belonging to the aligned noun + noun compounds. Translation template $N_{1}^{J} N_{2}^{J}$ $\rightarrow N_{1}^{C} N_{2}^{C}$ is used at 32 compounds and judged as the most frequently used template. And among those 32 compounds, there were 30 compounds have been translated successfully, the accuracy is around $93.8 \%$. For the translation template $N_{1}^{J} N_{2}^{J} \rightarrow N_{1}^{C} d e N_{2}^{C}$, the accuracy is only $66.7 \%$.
Table 5. Result of aligned case

\begin{tabular}{llccc}
\hline \hline No. & Template & Number & Success & Accuracy \\
\hline \hline 1. & $N_{1}^{J} N_{2}^{J} \rightarrow N_{1}^{C} N_{2}^{C}$ & 32 & 30 & $93.8 \%$ \\
\hline 2. & $N_{1}^{J} N_{2}^{J} \rightarrow N_{2}^{C} N_{1}^{C}$ & 15 & 12 & $80 \%$ \\
\hline 3. & $N_{1}^{J} N_{2}^{J} \rightarrow N_{1}^{C} d e N_{2}^{C}$ & 3 & 2 & $66.7 \%$ \\
\hline 4. & $N_{1}^{J} N_{2}^{J} \rightarrow N_{2}^{C} \operatorname{deN}_{1}^{C}$ & 1 & 1 & $100 \%$ \\
\hline 5. & $N_{1}^{J} N_{2}^{J} \rightarrow N_{2}^{C} l e N_{1}^{C}$ & 0 & 0 & - \\
\hline 6. & $N_{1}^{J} N_{2}^{J} \rightarrow N_{1}^{C} z h i N_{2}^{C}$ & 2 & 2 & $100 \%$ \\
\hline 7. & $N_{1}^{J} N_{2}^{J} \rightarrow \operatorname{cong} N_{1}^{C} N_{2}^{C}$ & 1 & 1 & $100 \%$ \\
\hline \hline Total & - & 54 & 48 & $88.9 \%$ \\
\hline \hline
\end{tabular}

Table 6. Examples of aligned case

\begin{tabular}{llll}
\hline \hline No. & Example & Translation & Other candidates \\
\hline \hline 1. & $\begin{array}{l}\text { keiyaku+syorui } \\
\text { (contract document) }\end{array}$ & hetong+shu & $\begin{array}{l}\text { qiyue+shu, } \\
\text { heyue+wenshu }\end{array}$ \\
\hline 2. & $\begin{array}{l}\text { kanri+kyouka } \\
\text { (strengthen } \\
\text { management) }\end{array}$ & jiaqiang+guanli & $\begin{array}{l}\text { qianghua+guanli, } \\
\text { zengqiang+guanli }\end{array}$ \\
\hline 3. & $\begin{array}{l}\text { keihi+zou } \\
\text { (increase of expense) }\end{array}$ & jingfei+de+zengjia & $\begin{array}{l}\text { feiyong+de+zengjia, } \\
\text { kaixiao+de+zengjia }\end{array}$ \\
\hline 4. & $\begin{array}{l}\text { hiyou+fudan } \\
\text { (expense burden) }\end{array}$ & fudan+de+feiyong & $\begin{array}{l}\text { chengdan+de } \\
+ \text { feiyong, fudan+de } \\
\text { +kaixiao }\end{array}$ \\
& & & - \\
\hline 5. & - & - & huiyuan+zhi+zhong, \\
\hline 6. & $\begin{array}{l}\text { kaiin+aida } \\
\text { (among members) }\end{array}$ & huiyuan+zhi+jian & \\
\hline 7. & $\begin{array}{l}\text { toujitsu+kisan } \\
\text { (calculating from }\end{array}$ & cong+dangtian & cong+dangri+qisuan, \\
& that day) & & cong+dangri+jisuan \\
\hline \hline
\end{tabular}

See Table 5 for details. Table 6 gives some detailed examples. No in Table 6 is indicated the translation template number. For each type of translation templates, we have given an example compound and the corresponding translation, and some candidates for understanding. The main reason for the failure of successful translation is failure of the correct selection.

(2) Partially Aligned: Not all component words of a compound noun are aligned. At some translation pairs, word correspondence is not observed at the either of the language or at the both of the languages. For the partially aligned alignment, it is still possible to get the corresponding translation according to the translation templates, but usually the translation is not the correct one. Such as, cyushin + syouhin (mainstay product) $\rightarrow$ zhizhu + chanpin, although zhizhu (mainstay) is not an exact translation of cyushin (center), but cyushin is lexically associated with Chinese zhizhu. Here 
Table 7. Examples of partially aligned case

\begin{tabular}{lll}
\hline \hline Example & Translation & Correct translation \\
\hline \hline $\begin{array}{l}\text { medama+syouhin } \\
\text { (a loss leader) }\end{array}$ & yanzhu+shangpin & remenhuo \\
\hline $\begin{array}{l}\text { unchin+syogakari } \\
\text { (sundry expenses) }\end{array}$ & yunfei+gezhong & gexiang+yunfei \\
\hline $\begin{array}{l}\text { cyushin+syouhin } \\
\text { (mainstay product) }\end{array}$ & $\begin{array}{l}\text { zhongxin } \\
\text { +shangpin }\end{array}$ & zhizhu+shangpin \\
\hline $\begin{array}{l}\text { syusei+keijyou } \\
\text { (modify budget) }\end{array}$ & xiuzheng+heji & xiugai+yusuan \\
\hline $\begin{array}{l}\text { syuryoku+syouhin } \\
\text { (a central product) }\end{array}$ & zhuli+shangpin & zhuda+shangpin \\
\hline $\begin{array}{l}\text { tocyuu+keika } \\
\text { (the situation of } \\
\text { progress) }\end{array}$ & tuzhong+jinzhan & jinzhan+qingkuang \\
\hline $\begin{array}{l}\text { keijyou+rieki } \\
\text { (ordinary profit) }\end{array}$ & pingjun+lirun & gujing+lirun \\
\hline $\begin{array}{l}\text { torihiki+jitsugyou } \\
\text { (the business results) }\end{array}$ & jiaoyi+shiye & shiji+jiaoyi+yeji \\
\hline $\begin{array}{l}\text { senpanr+rai } \\
\text { (for some time) }\end{array}$ & qianxie+rizi & qian+jitan \\
\hline $\begin{array}{l}\text { kinou+fuguai } \\
\text { (have some problems) }\end{array}$ & jineng+buquan & cunzai+de+wenti \\
\hline $\begin{array}{l}\text { hanmei+shidai } \\
\text { (as soon as thing } \\
\text { become clear) }\end{array}$ & $\begin{array}{l}\text { yi+nongqingchu } \\
\text { +jiu }\end{array}$ & $\begin{array}{l}\text { yi+diaocha+qing- } \\
\text { chu+yuanyin+jiu } \\
\text { (for private use) }\end{array}$ \\
\hline \hline
\end{tabular}

Table 8. Examples of unaligned case

\begin{tabular}{llc}
\hline \hline Example & Translation & Correct translation \\
\hline \hline $\begin{array}{l}\text { tenpu+fairu } \\
\text { (attached file) }\end{array}$ & tianfu+wenjian & fujian \\
\hline $\begin{array}{l}\text { senmon+uriba } \\
\text { (special counter) }\end{array}$ & zhuanmen+guitai & zhuangui \\
\hline $\begin{array}{l}\text { aramu+tokei } \\
\text { (alarm clock) }\end{array}$ & naozhong+biao & naozhong \\
\hline $\begin{array}{l}\text { teirei+kaigi } \\
\text { (a regular meeting) }\end{array}$ & dingli+huiyi & lihui \\
\hline $\begin{array}{l}\text { keiyaku+keizoku } \\
\text { (extending a contract) }\end{array}$ & hetong+jixu & xuyue \\
\hline \hline
\end{tabular}

the link becomes more tenuous. Some examples of partially aligned case are shown in Table 7 . Let us see the last example shown in the Table 7, through the translation template $N_{1}^{J} N_{2}^{J} \rightarrow N_{1}^{C} N_{2}^{C}$, we can get the translation of jika + you (for private use) which is zijia + yong. The literal meaning of the translation is correct. It has the same meaning with the standard translation jiating + xiaofei, under different expression. In such a case we also judge it as a unsuccessful one.

(3) Unaligned: No component words align between the Japanese compound noun and its translation. Typical of them is the case where the standard translation is a single word or when no word pair aligns. For example, keiyaku + keizoku (contract extension) $\rightarrow$ xuyue, mezamashi + tokei (alarm clock) $\rightarrow$ naozhong, teiri + kaigi (a regular meeting $) \rightarrow$ lihui. See detailed examples in Table 8 .

Except for aligned alignment, obtaining translation through the use of translation template is possible at the partially aligned and unaligned alignments. The reason we could not get the successful translation results is that the the compo- nent words of Japanese compound are partially or not aligned to their translations, so in those cases, the translation of the compound which we get from the translation templates usually is not a correct one.

(4) No Template Found: The translation does not correspond to any existed translation template. Such as: daitaihin + hojyuu (fill substitute) $\rightarrow$ yong + tidaipin + buchong, syufuku + cyuu (in restoration $) \rightarrow$ zhengzai + jinxing + xiuli, they are examples of misalignment due to missing templates.

The results of alignment are shown in Table 4. In the total 80 compound nouns, there is 54 compounds were classified as aligned translation pairs, and the coverage is around $67.5 \%$ which is the biggest one in those four cases. See the Table 5, we can see that for those 54 aligned translation pairs $48(88.9 \%)$ noun compounds have been translated correctly. The result shows that for those aligned noun compounds, the accuracy of translations is not very high for the case of aligned noun compounds. As the word level translation dictionary which is used currently, is still not a complete one, and therefore there will be many words still not accounted for. If we can use a more reliable word level translation dictionary, a high accuracy of translation will be expected.

It is found from the results that there is only 54 (67.5\%) noun compounds were classified as aligned noun compounds, we take attention to those misalignment in translation pairs. For misalignments which is caused by missing templates, it would be simply solved by extending corresponding translation templates to capture examples. For misalignments which is caused by the fact that the standard translation is a single word, explicit abbreviation expansion could unearth the full wordform and facilitate alignment. For those partially unaligned translation pairs, it might be possible to align words using word similarity, to translate some component of compounds. There are a number of avenues for dealing with those partially aligned and unaligned translation pairs in the course of efforts for the enhancement of the performance.

\section{Related Work}

There are several challenge involved in the automatic translation of compounds, and there are also quite different approaches suggested to deal with some of these issues. There are different views on the level of analysis required for proper translation of noun compounds, and correspondingly, the proposed strategies may generally be classified as either deep or shallow. In strategies based on deep processing, there is an attempt to semantically analyse the compounds, and the translation relation is defined via an intermediate representation.

Paggio and Qrsnes ${ }^{(15)}$ present a study on the feasibility of semantic analysis for translating Danish nominal compounds into Italian. As is common when dealing with compound interpretation, they distinguish between cases where correspondence between arguments are established and the cases the correspondence are not 
found. The head of an argumental compound is generally derived from a verb for which the non-head fills an argumental slot. According to Paggio and Qrsnes, the analysis of these will not impose major difficulties. They claim that there are clear preferences in sorts of arguments for filling of non-head position. They also claim that the preposition to generate will be determined by the valence restrictions of the Italian head-noun. Instead they focus on non-argumental compounds.

Navigli et al. ${ }^{(16)}$ also experiments with an interpretationdriven approach to the translation of English compounds to Italian, though they narrow the task to involve only complex terms from a limited domain. By using a machine-learned ontology, the parts of the source language compound are first disambiguated and mapped to concepts (WordNet synsets). To assign the correct relation holding between the compound parts, an inductive machine learning technique is used. The learned rules are based on triplets of concept-relation-concept, where each concept is represented by its set of WordNet hypernyms. Italian lexemes are then generated using Euro WordNet, and the conceptual relation is mapped to an Italian preposition by hand-crafted rules. Since there is not always a single mapping from a conceptual relation to a preposition, several suggestions will often be generated. The same of course holds for the synset translations. To select the best out of the generated candidates, Search is made at Google and the one with the most hits is chosen.

The above two approaches merely aim to solve the problem of detection of the appropriate construction type regarding the selection of preposition and to partially solve the problem of the choice of a relational adjective. They do not address the problems involved in the appropriate number and definiteness of the non-head nor any integrational issues.

Since a content expressed as a compound in one language may best be expressed by some other construction type in another language, the use of compounds cannot be solely explained in terms of semantic and pragmatic factors, but is in part a question of language-specific usage. For those shallow approaches, the strategies proposed for compound translation are mainly based on the use of monolingual corpora. These are easier to be obtained in very large sizes thus alleviating the problem of data sparseness.

Lee and Kim $(2002)^{(5)}$ use definitions from a bilingual dictionary and target language corpora, and treat translation selection as disambiguation of a source word sense and selection of a target word. They report the accuracy of their method for nouns to be 0.55 , although in the case of compound nouns it would be overkilling to rely on such rich semantic resources to achieve relatively modest precision.

Rackow et al. (1992) ${ }^{(17)}$ look at a German-English compound noun MT task over a range of translation templates. They propose the use of "default constructions" for single words in a manner similar to that described in this research, but base determination of such defaults on English translations within a parallel corpus.
For example, they take the Hansards corpus and observe which of "ecology" and "ecological" occurs more often as a noun premodifier and use this information in generating ecological movement rather than ecology movement as a translation for the German Umweltbewegung. Unfortunately, no attempt is made to evaluate the method.

Grefenstette (1999) ${ }^{(18)}$ uses web data to select English translations for compositional German and Spanish noun compounds, and achieves an impressive accuracy of 0.86-0.87. The translation task Grefenstette targets is intrinsically simpler than that described in this paper, however, in that he filters out the effects of translation template selection by considering only those compounds which is translated into NN compounds in English. It is also possible that the historical relatedness of languages has an effect on the difficulty of the translation task, although further research would be required to confirm this prediction. Having said this, the successful use of web data by a variety of researchers suggests an avenue for future research in comparing our results with those obtained using web data.

Following the strategy proposed in Tanaka $(2002)^{(6)}$, the best translation candidate is not assumed to correspond to the most frequently occurring candidate in a target language corpus, but rather to the candidate that appears in the contexts nearest to that of the source language compound. In practice, this strategy provides a modification of the selection phase. Sets of translation candidates are first generated by translating the source language lexemes word-by-word, slotting these into templates and then keeping only these combinations that are attested in a target language corpus. Note that this strategy only accounts for translations that are attested as wholes, and that it thereby will have difficulties translating completely novel compounds. Word translations are quite generously defined; source and target lexemes need not be linked via a dictionary. To select the most plausible translation, the context of source language instances in a mono-lingual corpus is compared to those of the candidate translations in a target language corpus. Context is defined by words co-occurring in the same sentence, and is represented by vectors where each position defines the relative relevance of a word given the compound ${ }^{\dagger}$ Source language vectors are converted to target language equivalents by multiplication with a predefined translation matrix. An evaluation of the selection method shows that the best translation candidate was chosen in more than $70 \%$ of the cases. In comparison to a baseline based on frequencies, the results proved to be significantly better.

\section{Conclusion and Future Work}

In order to get correct translations of noun compounds to enhance the performance of Japanese-Chinese MT system SFBMT, we proposed in this paper a shallow method based on a word level translation dictionary and target language monolingual corpus for translating noun

$\dagger$ The relative relevance of the co-occurring words is calculated by a log-likelihood ratio as defined by Dunning (1993) ${ }^{(19)}$. 
compounds. Our method showed that it can act as a standalone module in SFBMT. The results of our experiments show that the method, if improved can perform the task of automatic compound noun translation quite well. First of all, a more reliable word level translation dictionary is required. Furthermore, if more trustworthy translation templates are extracted automatically, the proposed method will provide far better result.

In future work, we would like to extend our experiment to larger corpus and as an improvement to the current method, we may consider the inclusion of semantic information for the resolution of some cases of noun compounds.

\section{Acknowledgment}

This research has been partially supported by the Ministry of Education, Culture, Sports, Science and Technology of Japan under Grant-in-Aid for Scientific Research (B), 14380166, 17300065, Exploratory Research, 17656128, and the Outstanding Overseas Chinese Scholars Fund of the Chinese Academy of Sciences (No.2003-1-1). We would also like to give thanks to the reviewers for their valuable comments.

(Manuscript received March 30, 2005,

revised Jan. 10, 2006)

\section{References}

(1) T. Tanaka and T. Baldwin: "Noun-Noun Compound Machine Translation: A Feasibility Study on Shally Processing", Proc. the ACL-2003 Workshop on Multiword Expressions: Analysis, Acquisition and Treatment, pp.17-24, Sapporo, Japan (2003)

( 2 ) P. Fung and K. McKeown: "Finding terminology translations from non-parallel corpora", Proc. the 5th Annual Workshop on Very Large Corpora, pp.192-202, Hong Kong (1997)

(3) R. Rapp: "Automatic identification of word translations from unrelated English and German corpora", Proc. the 37th Annual Meeting of the ACL, pp.1-7, College Park, USA (1999)

(4) T. Tanaka and Y. Matsuo: "Extraction of translation equivalents from non-parallel corpora", Proc. the 8th International Conference on Theoretical and Methodological Issues in Machine Translation (TMI-99), pp.109-119, Chester, UK (1999)

(5) H.A. Lee and G.C. Kim: "Translation selection through source word sense disambiguation and target word selection", Proc. the 19th International Conference on Computational Linguistics (COLING2002), pp.530-536, Taipei, Taiwan (2002)

(6) T. Tanaka: "Measuring the similarity between compound nouns in different languages using non-parallel corpora", Proc. the 19th International Conference on Computational Linguistics (COLING 2002), pp.981-987, Taipei, Taiwan (2002)

( 7 ) X. Zhao, F. Ren, and S. Voß: "A Super-Function Based Japanese-Chinese Machine Translation System for Business Users", The 6th Conference of the Association for Machine Translation in the Americas (2004)

(8) F. Ren: "Super-function based machine translation", Communications of COLIPS, Vol.9, No.1, pp.83-100 (1999)

(9) X. Zhao, F. Ren, S. Kuroiwa, and M. Sasayama: "JapaneseChinese machine translation system using SFBMT", Proc. the Second International Conference on Information, pp.16-21, Beijing (2002)

(10) http://www.jusnet.co.jp/business/bunrei.html.June 2004 (date of last check).

(11) P. Koehn and K. Knight: "Feature-rich statistical translation of noun phrases", Proc. the 41st Annual Meeting of the ACL, pp.311-318, Sapporo, Japan (2003)

(12) T. Tanaka and T. Baldwin: "Translation selection for Japanese-English noun-noun compounds", Proc. the Ninth Machine Translation Summit (MT SummitIX), pp.89-96, New Orleans, USA (2003)
(13) K. Maruyama, M. Doi, Y. Iguchi, K. Kuwabara, M. Onuma, T. Yasui, and R. Yokosuka: "Writing Business Letters In Japanese", Original Japanese edition published by The Japan Times, Ltd. (2003)

(14) K. Fujimoto, L. Zhang, and H. ShiYun: "Chinese-Japanese Business Letters Encyclopedia", TOHU Shoten 1(995)

(15) P, Patrizia and B. Orsnes: "Automatic Translation of Nominal Compounds - A Case Study of Danish and Italian", Rivista di Linguistica, Vol.5, No.1, Rosenberg \& Sellier, Torino (1994)

(16) R. Navigli, P. Velardi, and A. Gangemi: "Ontology Learning and its application to automated terminology translation", IEEE Intelligent Systems (2003)

(17) U. Rackow, I Dagan, and U. Schwall: "Automatic translation of noun compounds", Proc. the 14th International Conference on Computational Linguistics (COLING1992), pp.1249-1253, Nantes, Frances (1992)

(18) G. Grefenstette: "TheWorldWideWeb as a resource for example-based machine translation tasks", Trans. and the Comput. 21: ASLIB'99, London, UK (1999)

(19) T. E. Dunning: "Accurate Methods for the Statistics of Surprise and Coincidence", Computational Linguistics, Vol.19, No.1, pp.61-74 (1993)

Xin Zhao (Non-member) was born in 1976. She received

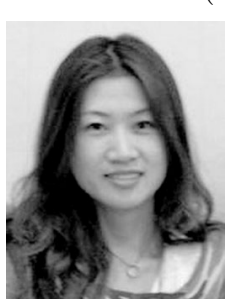
the B.E degree in 1998 from LiaoNing University, ShenYang, LiaoNing P.R. China, received M.E in 2002 from The University of Tokushima, Tokushima, Japan. She worked at NEU-BITCO DaLian, LiaoNing P.R. China from 1998 to 2000. Her current research interests include machine translation, natural language processing, and artificial intelligence.

Fuji Ren (Non-member) received the Ph.D. degree in 1991

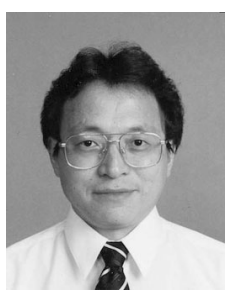
from Faculty of Engineering Hokkaido University, Sapporo, Japan. He worked at CSK, Tokyo, Japan, where he was a chief researcher of NLP. 1994 to 2001, he was an Associate Professor in the Faculty of Information Sciences, Hiroshima City University. From 2001, he joined the Faculty of Engineering, the University of Tokushima, Japan, as a Professor. He was a visiting professor at CRL Computing Research Laboratory, New Mexico State University, USA, from 1996-1997. His current research interests include natural language processing, machine translation, artificial intelligence, and MultiLingual Multi-Function Multi-Media Intelligent System.

Singo Kuroiwa (Member) received the B.E., M.E. and D.E.

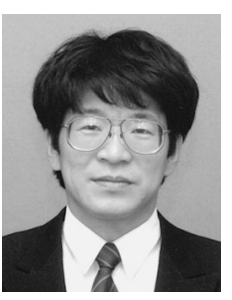
degrees in electro-communications from The University of Electro Communications, Tokyo, Japan, in 1986, 1988, and 2000 respectively. From 1988 to 2001 he had been a researcher at the KDD R \& D Laboratories. Since 2001, he has been with the Faculty of Engineering, Tokushima University, Tokushima, Japan, where he is currently an Associate Professor. $\mathrm{He}$ is a member of the Information Processing Society, the Acoustical Society of Japan, and The Institute of Electronics, Information and Communication Engineers. His current research interests include speech recognition, natural language processing, and information retrieval. 